\title{
Simulating Focused Ultrasound Transducers using Discrete Sources on Regular Cartesian Grids
}

\author{
Eleanor Martin, Yan To Ling, and Bradley E. Treeby
}

\begin{abstract}
Accurately representing the behaviour of acoustic sources is an important part of ultrasound simulation. This is particularly challenging in ultrasound therapy where multielement arrays are often used. Typically, sources are defined as a boundary condition over a $2 \mathrm{D}$ plane within the computational model. However, this approach can become difficult to apply to arrays with multiple elements distributed over a non-planar surface. In this work, a grid-based discrete source model for single and multi-element bowl-shaped transducers is developed to model the source geometry explicitly within a regular Cartesian grid. For each element, the source model is defined as a symmetric, simply-connected surface with a single grid point thickness. Simulations using the source model with the opensource k-Wave toolbox are validated using the Rayleigh integral, O'Neil's solution, and experimental measurements of a focused bowl transducer under both quasi continuous wave and pulsed excitation. Close agreement is shown between the discrete bowl model and the axial pressure predicted by O'Neil's solution for a uniform curved radiator, even at very low grid resolutions. Excellent agreement is also shown between the discrete bowl model and experimental measurements. To accurately reproduce the near-field pressure measured experimentally, it is necessary to derive the drive signal at each grid point of the bowl model directly using holography. However, good agreement is also obtained in the focal region using uniformly radiating monopole sources distributed over the bowl surface. This allows the response of multi-element transducers to be modelled, even where measurement of an input plane is not possible.
\end{abstract}

\section{INTRODUCTION}

A CCURATELY representing source conditions is an important part of numerical simulation in ultrasound, particularly in high-intensity focused ultrasound (HIFU) and other ultrasound therapies where multi-element arrays are used $[1,2]$. Typically, ultrasound sources are defined within computational models as boundary conditions defined over a 2D input plane [3]. These input planes are either measured experimentally $[2,4]$, or projected using analytical expressions [5]. However, for arrays with multiple elements distributed over a non-planar surface, there are two limitations with this approach. First, if the focal region is close to or within the bounding surface of the array (as is the case for hemispherical arrays used in transcranial applications [6]), it is difficult to define a single 2D plane over which the source can be measured and applied. Second, the input plane

Manuscript received XX; revised XX. This work was supported by the Engineering and Physical Sciences Research Council (EPSRC), UK, grant numbers EP/L020262/1 and EP/M011119/1.

The authors are with the Department of Medical Physics and Biomedical Engineering, University College London, London, UK (email: elly.martin@ucl.ac.uk). Y. Ling is currently with the Interdisciplinary Division of Biomedical Engineering, The Hong Kong Polytechnic University, Hong Kong, China..

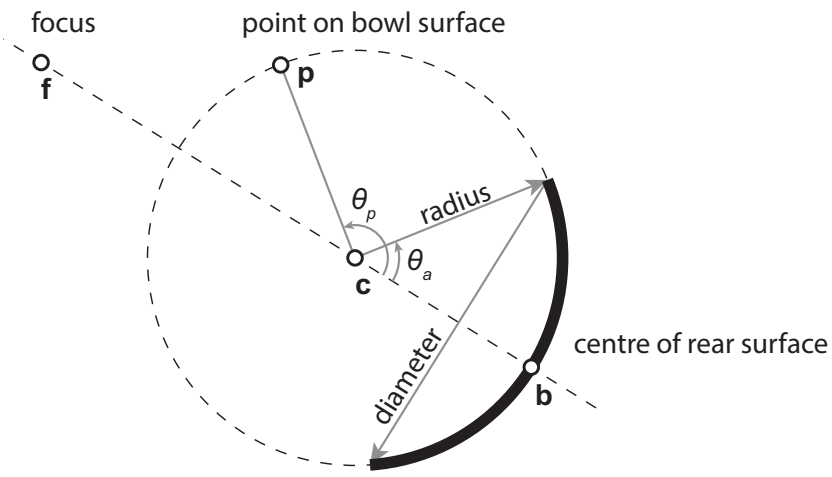

Fig. 1. Geometry of the discrete source model. Each bowl transducer is defined by the position of the rear surface $\mathbf{b}$, the radius of curvature, the diameter, and a point on the beam axis $\mathbf{f}$.

must be defined (i.e., measured or projected) for each set of drive conditions. This is a significant limitation when investigating the response of clinical HIFU systems, which may have hundreds or thousands of individual elements that are phased differently for each sonication [2]. One way in which these limitations can be overcome is to use an explicit source model. In this case, the response of the source is included within the model as the injection of mass or force at particular grid points within the computational mesh, rather than the imposition of a planar boundary condition. For finite difference and pseudospectral models, which are arguably the most commonly used numerical methods in ultrasound simulation [7], a regular Cartesian grid is generally used, and thus the source geometry must also conform to this mesh. In this work, a grid-based discrete source model for single and multi-element bowl-shaped transducers is developed, and validated against analytical models and experimental data.

\section{Discrete Source Model}

\section{A. Requirements}

There are several requirements for the grid-based bowlshaped source model. First, sources of the same radius of curvature and diameter facing the positive/negative $\mathrm{x}$-direction, $\mathrm{y}$-direction, or z-direction should be represented by the same shape and the same number of grid points. This means the sphere on which the bowl lies should have three axes of ordertwo rotational symmetry. Second, the source model should be simply connected and have only a single grid point thickness. This is to ensure that the acoustic field generated by the source is neither magnified or smoothed due to overlapping source points nor reduced due to discontinuities. The model is simply 

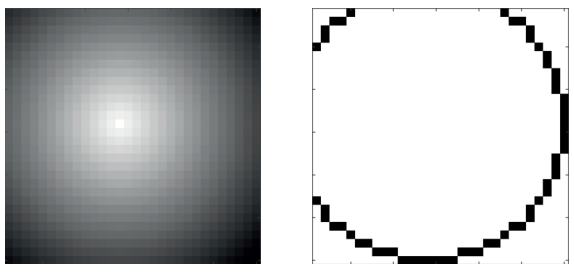

(b) Circle/Sphere

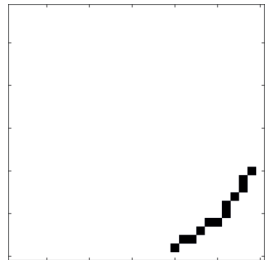

(c) Arc/Bowl

Fig. 2. Two-dimensional illustration of the steps used to create the discrete source model. First, a distance matrix is calculated containing the Euclidean distance from each point to the centre of the sphere on which the bowl lies (darker indicates larger distance). Next, the grid points where the distance is within 0.5 grid steps of the radius of curvature are labelled. Finally, points that lie outside the arc angle of the bowl are removed.

connected if for each grid point marked as part of the source, exactly 8 out of the 26 neighbouring points are also marked (with the exception of grid points along the outer rim of the source). In this context, a surface defined within the Cartesian grid is understood to be a collection of simply connected grid points.

\section{B. Formulation}

The geometry of the bowl-shaped source model is defined as shown in Fig. 1 (the bowl is mathematically equivalent to a spherical cap). Each transducer element is defined by the position of the centre of the rear surface of the bowl $b$ (analogous to the midpoint of an arc in 2D), the radius of curvature of the bowl, the diameter, any point on the beam axis $\mathbf{f}$ where $\mathbf{f} \neq \mathbf{b}$ (this defines the orientation of the source), and the overall size of the Cartesian grid in which the source is defined. Using these parameters, the position $\mathbf{c}$ of the centre of the sphere on which the bowl lies is calculated using

$$
\mathbf{c}=\frac{\text { radius }}{\|\mathbf{f}-\mathbf{b}\|}(\mathbf{f}-\mathbf{b})+\mathbf{b} \text {. }
$$

A distance matrix is then created which contains the Euclidean distance from each point in the Cartesian grid to $\mathbf{c}$. An example in 2D is given in Fig. 2(a). A series of bi-directional line searches along each dimension of the distance matrix are then conducted (i.e., first along all the rows in each direction, then along the columns, etc). The grid points with Euclidean distance to the sphere centre within 0.5 grid steps of the radius of curvature are then labelled. This results in a singly connected sphere (or circle in 2D) with the correct radius centred at c. An example is shown in Fig. 2(b).

Next, the grid points within the sphere that do not form part of the bowl are removed. This is performed by calculating the angle $\theta_{p}$ between the vector from each grid point $\mathbf{p}$ on the sphere surface to the sphere centre $\mathbf{c}$, and the vector from the rear surface of the bowl $\mathbf{b}$ to the sphere centre using the geometric definition of the dot product

$$
\theta_{p}=\cos ^{-1}\left(\frac{(\mathbf{p}-\mathbf{c}) \bullet(\mathbf{b}-\mathbf{c})}{\|\mathbf{p}-\mathbf{c}\|\|\mathbf{b}-\mathbf{c}\|}\right) .
$$

The grid points for which $\theta_{p}$ is greater than the half arc angle $\theta_{a}$, where

$$
\theta_{a}=\sin ^{-1}(\text { diameter } /(2 \times \text { radius })),
$$
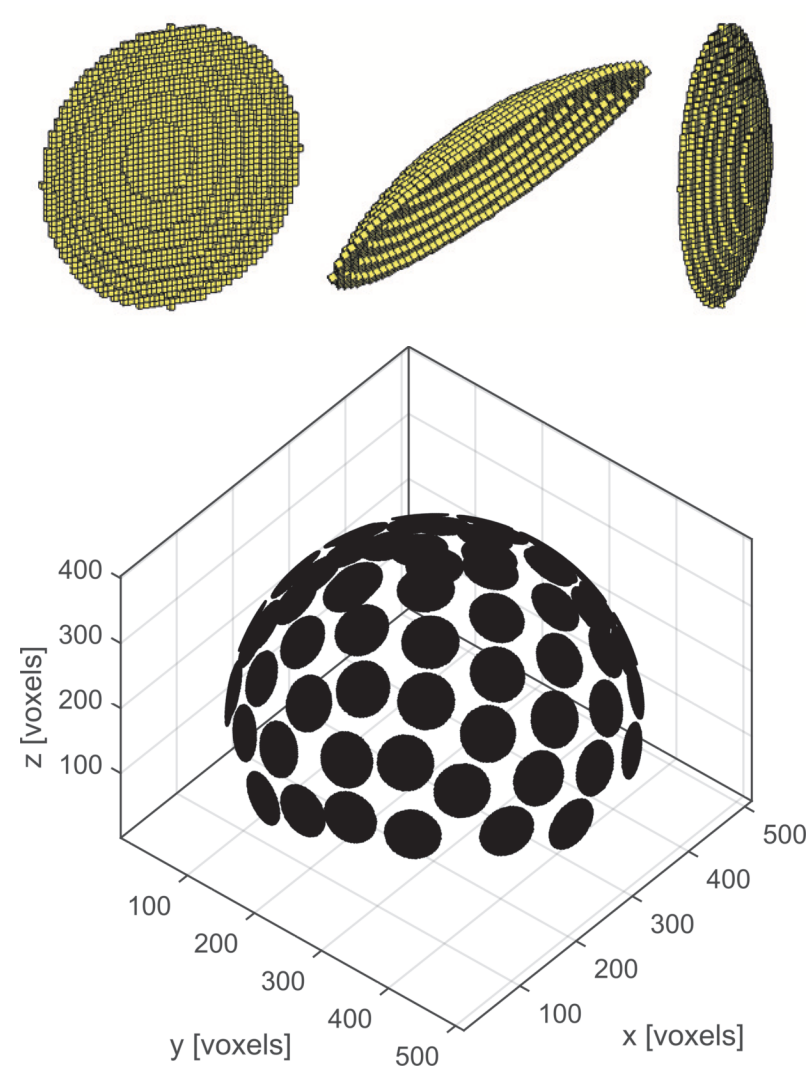

Fig. 3. (Top) Three views of a low resolution grid-based bowl transducer generated as described in Sec. II.B. The radius of curvature of the bowl is 100 grid points, and the diameter is 45 grid points. (Bottom) Example of a multi-element hemispherical transducer array containing 64 individual bowls.

are then removed. This leaves a symmetric and simplyconnected bowl-shaped surface with a single grid point thickness. Functions to generate single and multiple bowls (in 3D) and single and multiple arcs (in 2D) using this approach were written in MATLAB. An example of a low resolution bowl and a multi-element hemispherical array containing 64 individual bowls (based on [6]) are shown in Fig. 3. Note, in Fig. 2 and Fig. 3, grid points that form part of the source are visually represented by pixels of size $\Delta x \times \Delta y$ (in 2D) or voxels of size $\Delta x \times \Delta y \times \Delta z$ (in 3D) centred about the position of each grid point.

\section{Source Scaling}

The representation of the bowl surface as a series of discrete grid points on the Cartesian grid gives rise to staircasing errors $[8,9]$. In particular, for low grid resolutions, the discrete source points are further away from the ideal bowl, which can affect the structure of the generated acoustic waves, particularly in the near-field. The staircased representation of the source geometry also introduces a scaling issue due to the different density of grid points in grid and diagonal directions. For example, a horizontal line tilted at $45^{\circ}$ to the grid axis will contain a factor of $\sqrt{2}$ fewer grid points per unit length than a horizontal line aligned with the grid axis. This disparity results in the generated acoustic pressure being a factor of $\sqrt{2}$ smaller. This can be corrected for by introducing an individual scaling factor for each grid point that forms part of the source. 
The scaling factor is calculated based on the average distance from the grid point to its connected neighbours, divided by the equivalent average distance for a reference plane (3D) or line (2D) source aligned with the Cartesian grid axes. For example, for a horizontal line tilted at $45^{\circ}$ to the grid axis, the average distance for a grid point on the line to its connected neighbours is $(\sqrt{2}+\sqrt{2}) / 2=\sqrt{2}$, while the reference distance is $(1+1) / 2=1$, giving a scaling factor of $\sqrt{2}$. As an alternative, it is also possible to extract a single scaling factor that corrects for the difference in peak pressure based on a reference simulation or measurement (i.e., normalise the maximum pressure to a known value). However, this factor will change depending on the discretisation parameters (see Fig. 4(c)), and requires a suitable reference value.

\section{NUMERICAL TESTING}

To test the grid-based discrete bowl model, a series of simulations were performed using the open source $\mathrm{k}$-Wave MATLAB toolbox $[10,11]$. This solves the acoustic equations on a regular Cartesian grid using a $k$-space pseudospectral scheme. The source was modelled as the injection of mass in free-space. Within $\mathrm{k}$-Wave, this is performed by defining a source mask (which specifies the grid points that form part of the source geometry), and a time varying source pressure for each grid point in the source mask. Further details about the governing equations and source terms can be found in Ref. [12] and the k-Wave user manual. Note that the solution obtained with a uniform mass or uniform monopole source distribution within k-Wave is equivalent to the Rayleigh and O'Neil integral solutions with a uniform distribution of the normal component of vibrational velocity over the transducer surface [7, 13].

The source geometry was based on the H-101 spherically focused single element HIFU transducer (Sonic Concepts, WA, USA). This has a nominal diameter of $64 \mathrm{~mm}$, and radius of curvature of $63.2 \mathrm{~mm}$. The discrete bowl surface was calculated as described in Sec. II.B, and used to define the source mask within $\mathrm{k}$-Wave. The beam axis was aligned with the Cartesian grid, and the source was driven by a continuous wave sinusoid at $1.1 \mathrm{MHz}$ assuming linear and lossless propagation. The medium properties were set to those of water at room temperature, with a sound speed of $1482 \mathrm{~m} / \mathrm{s}$ and density of $998.2 \mathrm{~kg} / \mathrm{m}^{3}$. For each simulation, the steady state pressure amplitude was recorded, and the $l_{2}$ relative error norm in the axial pressure was calculated according to

$$
l_{2}[\%]=100 \times \sqrt{\frac{\sum_{x}\left(p_{\text {kwave }}(x)-p_{\text {ref }}(x)\right)^{2}}{\sum_{x}\left(p_{\text {ref }}(x)\right)^{2}}} .
$$

The reference axial pressure was calculated using the O'Neil solution for a focused radiator of the same dimensions [15].

Simulations were repeated using grid discretisations from 2.2 points per wavelength (PPW) up to $11.9 \mathrm{PPW}$. This corresponds to grid sizes of $256 \times 128 \times 128$ up to 1408 $\times 704 \times 704$ grid points. The simulations were run using the parallelised $\mathrm{C}++$ version of $\mathrm{k}$-Wave [12]. The numerical model used in $\mathrm{k}$-Wave is exact in the limit of wave propagation in a homogeneous and lossless medium [10]. This means
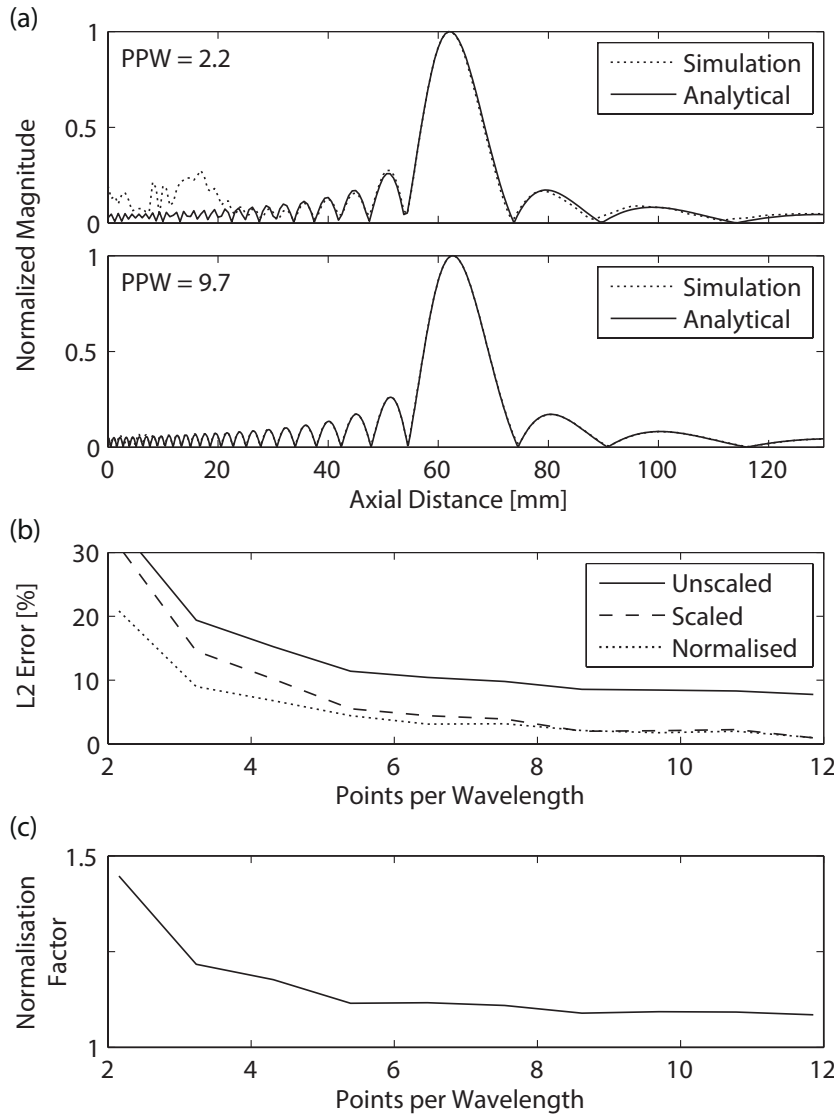

Fig. 4. (a) Axial pressure from a focused bowl transducer calculated using the O'Neil model (solid lines) and the discrete source model (dotted lines) for two different points per wavelength (PPW). Even at very coarse grid resolutions close to the Nyquist limit, the main features of the pressure field are captured. (b) Convergence of the $l_{2}$ relative error norms with the number of PPW. (c) Normalisation factor between the unscaled and analytical maximum pressure.

changing the number of PPW only changes the discretisation of the source geometry, and not the accuracy of the numerical model. (While the numerical model can theoretically propagate waves up to the Nyquist limit of 2 PPW, in practice, a slightly higher PPW is needed for the perfectly matched layer to work correctly [14].)

Results for the on-axis pressure for 2.2 and 9.7 PPW are shown in Fig. 4(a). Even at very low PPW close to the Nyquist limit, the main features of the beam are still captured by the model. The biggest discrepancies are in the near-field region close to the source. This is due to the spatial offset between the desired bowl geometry and the discrete set of grid points used to represent it. This offset may be up to half a grid step (which corresponds to a quarter of a wavelength at 2 PPW), and has a noticeable effect on the near-field pressure distribution. It may be possible to reduce this error at low PPW by introducing a phase shift or time delay for each discrete source point dependent on its distance from the desired geometry.

At higher PPW, there is very close agreement between the simulation and analytical results. The convergence of the $l_{2}$ relative error norm with the number of PPW is shown in Fig. 4(b). The three curves correspond to the source with no scaling (solid line), the source with individual scaling factors as described in Sec. II-C (dashed line), and the unscaled source 
normalised to the maximum pressure of the O'Neil solution (dotted line). As expected, the error in the unscaled source does not approach zero at higher PPW due to staircasing errors. However, when the source is normalised or the source scaling is included, the errors converge very rapidly. The error is below $5 \%$ by $6 \mathrm{PPW}$, and below $1 \%$ by $12 \mathrm{PPW}$. The normalisation factor for the unscaled source is shown in Fig. 4(c). At low PPW, this factor has a strong dependence on the grid parameters. Consequently, a normalisation factor calculated using one value of PPW should not be used at other values of PPW or for other bowl geometries.

\section{EXPERIMENTAL TESTING}

\section{A. Experimental Measurements}

In addition to numerical testing, simulations using the source model were compared with experimental measurements made in the acoustic field generated by a single element spherically focused HIFU transducer (H-101, Sonic Concepts, as above). The acoustic pressure field was measured in a tank of deionized water (at $22 \pm 0.1{ }^{\circ} \mathrm{C}$ ) with a $0.2 \mathrm{~mm}$ PVDF needle hydrophone (Precision Acoustics, Dorchester, UK) as shown in Fig. 5. The hydrophone was positioned using a scanning tank (Precision Acoustics, Dorchester, UK) with five computer controlled translation stages. The transducer was driven by a signal generator (33522A, Agilent Technologies, Santa Clara, CA, USA) connected via a $75 \mathrm{~W}$ power amplifier (A075, E\&I, Rochester, NY, USA) and impedance matching network.

Measurements were made under both quasi continuous wave and pulsed conditions. In the quasi continuous wave case, the transducer was excited by a 45 cycle sinusoidal burst at a frequency of $1.1 \mathrm{MHz}$ and a pulse repetition frequency of $200 \mathrm{~Hz}$. To achieve steady state conditions, the hydrophone signal was acquired in a time window which occurred after signals from all parts of the transducer had arrived but before reflections from the measurement equipment had reached the hydrophone. A planar scan perpendicular to the beam axis was acquired at an RMS drive voltage of $2.7 \mathrm{~V}$ (measured at the matching network). Additional axial line scans were performed at both this drive level and at an RMS voltage of $5.4 \mathrm{~V}$.

In the pulsed wave case, the transducer was excited by a 4 cycle sinusoidal pulse at a frequency of $1.1 \mathrm{MHz}$ and a pulse repetition frequency of $200 \mathrm{~Hz}$. The time window for acquisition of the hydrophone signal included the earliest and latest arrival times of the pulse from all parts of the transducer. A planar scan perpendicular to the beam axis was acquired at a peak-to-peak drive voltage of $7.3 \mathrm{~V}$. Additional axial line scans were performed at this drive level and at a peak-topeak voltage of $16.6 \mathrm{~V}$. In both cases, low transducer drive levels were used to allow the use of a conventional PVDF hydrophone for measurement of the acoustic field.

In both the continuous wave and pulsed conditions, the planar scan covered an area of $45 \mathrm{~mm} \times 45 \mathrm{~mm}$ at a distance of $42.5 \mathrm{~mm}$ from the rear surface of the transducer with a spatial step size of $300 \mu \mathrm{m}$. Signals were digitised with an Tektronix DPO5034B Digital Phosphor Oscilloscope (Tektronix, UK Ltd., Berkshire, UK) with a sampling frequency of 125

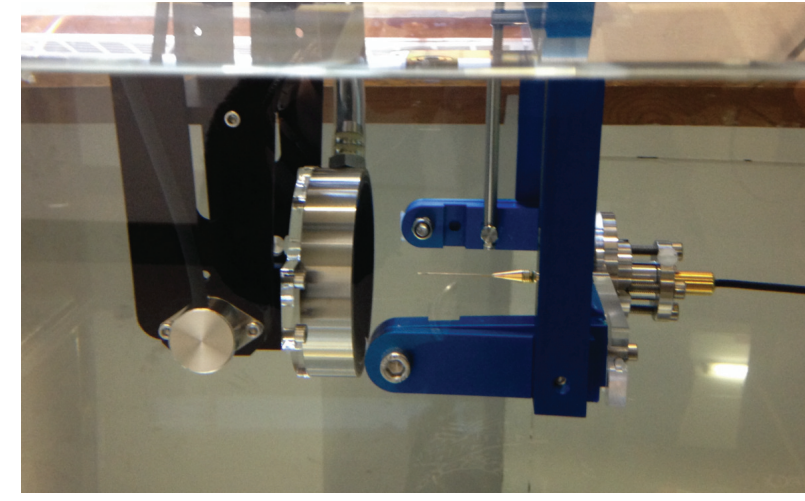

Fig. 5. Photograph of the experimental setup showing the bowl-shaped H101 transducer and the $0.2 \mathrm{~mm}$ PVDF needle hydrophone mounted in the scanning tank.

$\mathrm{MHz}$ and 32 averages. Line scans were performed along the transducer beam axis from a distance of $30 \mathrm{~mm}$ to $100 \mathrm{~mm}$, with a step size of $200 \mu \mathrm{m}$.

\section{B. Simulation Setup and Optimisation of Bowl Geometry}

The experimental data from the planar scans was used to obtain the pressure at the surface of the discrete bowl source using linear acoustic holography. k-Wave simulations (including absorption and nonlinearity) based on the discrete bowl model were then performed to obtain the generated pressure field. For the simulations, the medium properties were set to those of water at $22{ }^{\circ} \mathrm{C}$ (the mean temperature during the measurements), with a sound speed of $1488.5 \mathrm{~m} / \mathrm{s}$, density of $997.8 \mathrm{~kg} / \mathrm{m}^{3}$, nonlinearity parameter (B/A) of 4.96 , and absorption coefficient of $2.17 \times 10^{-3} \mathrm{~dB} \mathrm{MHz}^{-2} \mathrm{~cm}$. The drive signals were defined in two ways: (1) directly using the pressure signal at each grid point of the bowl model calculated using holography, and (2) using a uniform mass source distribution across the transducer surface. While the resulting pressure distribution at the source in this case is not necessarily uniform, for convenience this condition will be herein referred to by the equivalent mean pressure amplitude, $\bar{p}_{0}$. As the source may not behave like an ideal radiator and the geometry may differ from the nominal parameters specified by the manufacturer, an optimisation was first performed to find the effective diameter and radius of curvature of the source. The optimisation was performed by minimising the $l_{2}$ error between the normalised pressure magnitude calculated using the Rayleigh integral for a focused radiator (calculated with a uniform distribution of the normal component of the vibrational velocity) and the normalised pressure magnitude measured on lines passing through the focus of the field in the axial and transverse directions. The obtained parameters were $61.7 \mathrm{~mm}$ for the transducer diameter and $63.2 \mathrm{~mm}$ for the radius of curvature.

\section{Continuous Wave Conditions}

For the continuous wave experiments, the magnitude and phase at $1.1 \mathrm{MHz}$ were extracted from the measured time domain pressure signals. The Rayleigh integral was then used 
(a)
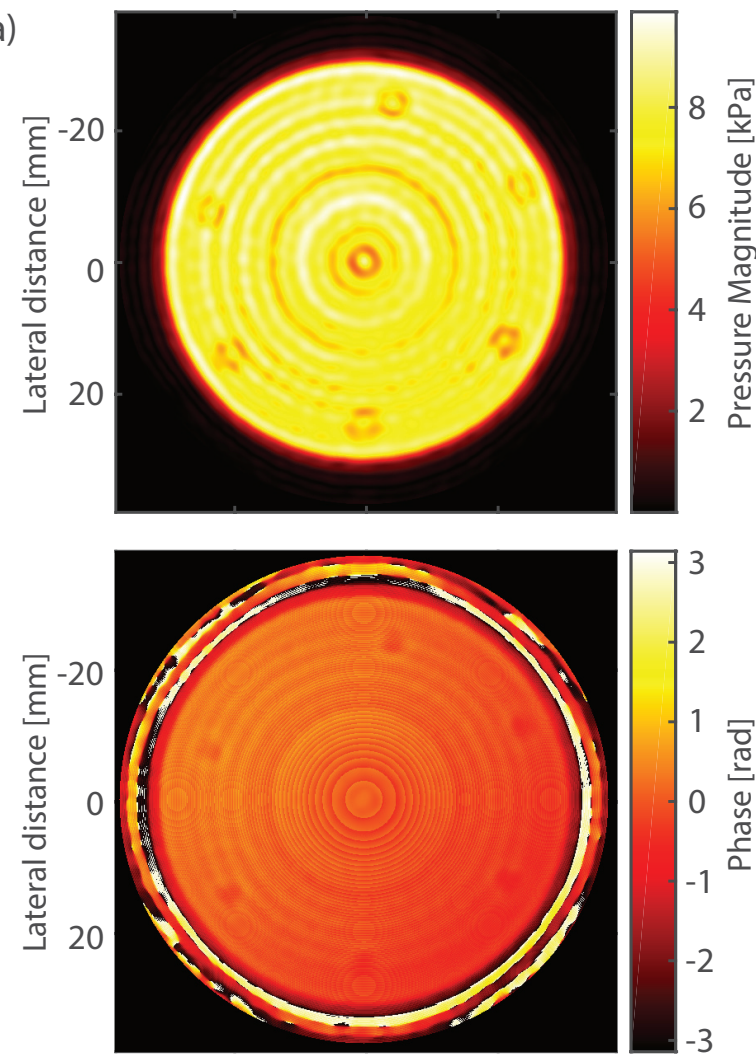

(b)

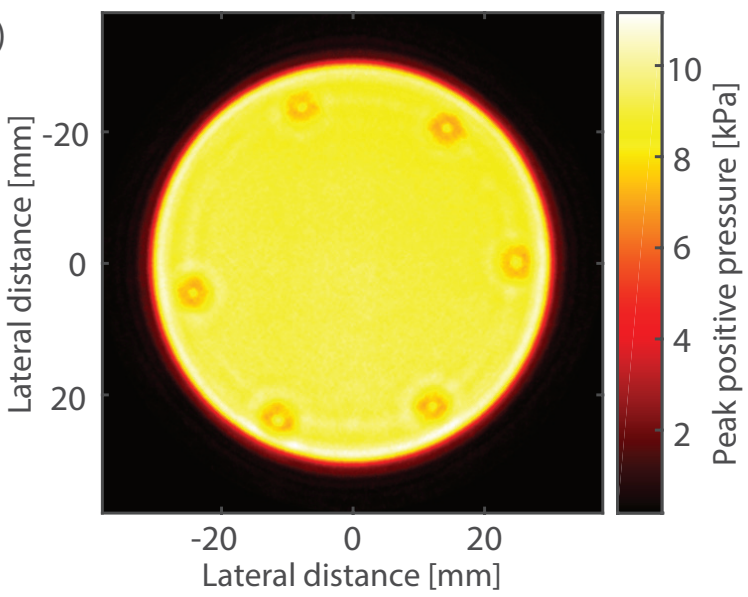

Fig. 6. (a) Magnitude and phase of the steady state source pressure on the surface of the bowl in continuous wave $(\mathrm{CW})$ mode reconstructed from measurements by back projection using the Rayleigh integral. The periodic structure in the magnitude plot is due to the presence of Lamb waves. The small variations in the phase plot are due to the staircased source geometry on the regular Cartesian grid. (b) Temporal peak positive source pressure on the surface of the bowl in pulsed wave (PW) mode reconstructed from measurements by back projection using k-Wave. Note, the PW source pressure appears rotated relative to the $\mathrm{CW}$ source pressure due to remounting of the transducer between measurements.

to calculate the complex pressure at the location of each grid point forming the bowl surface. In order to fully capture the source pressure distribution, the pressure was also calculated over an area outside the bowl equal to approximately $20 \%$ of the bowl diameter. The source hologram is shown in Fig. 6(a). In the first set of simulations, the magnitude and phase at each grid point over the bowl surface was used to create a set of

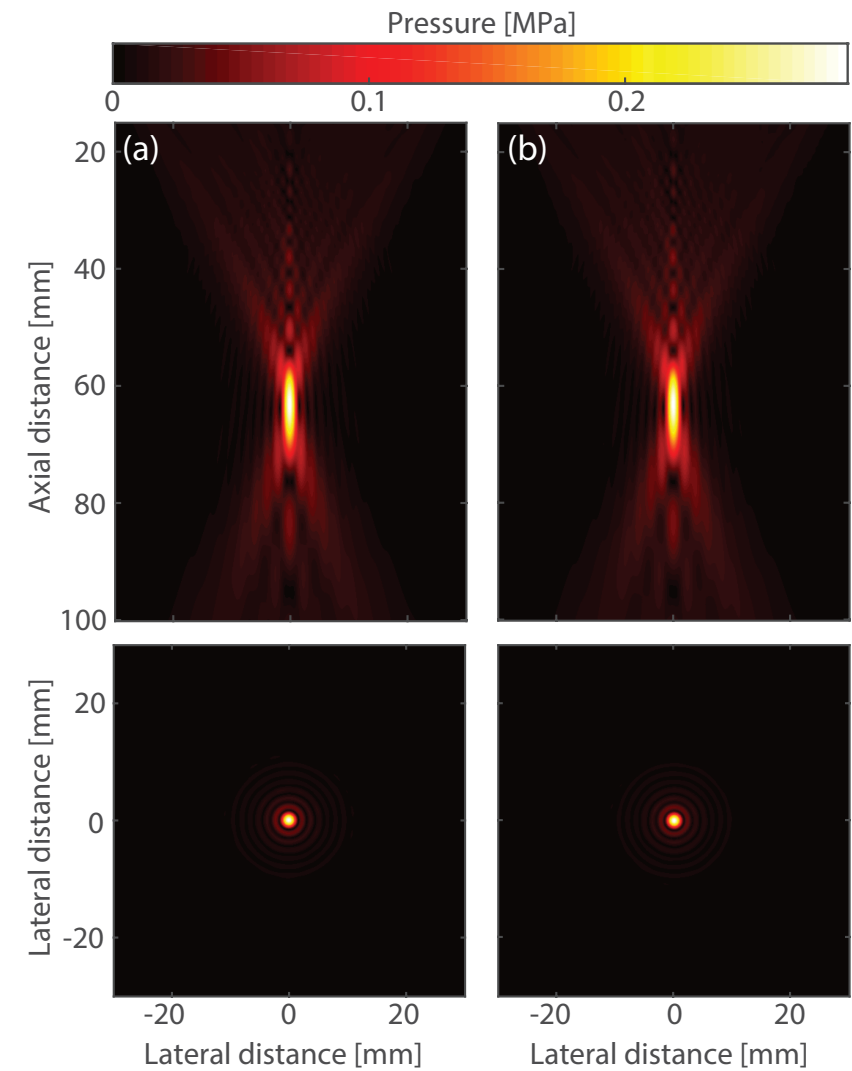

Fig. 7. Comparison of pressure calculated on axial and lateral planes through the focus under continuous wave conditions using (a) the Rayleigh integral with the source hologram as the input pressure, and (b) k-Wave with the discrete bowl model as the source geometry and the source hologram as the input source pressure.

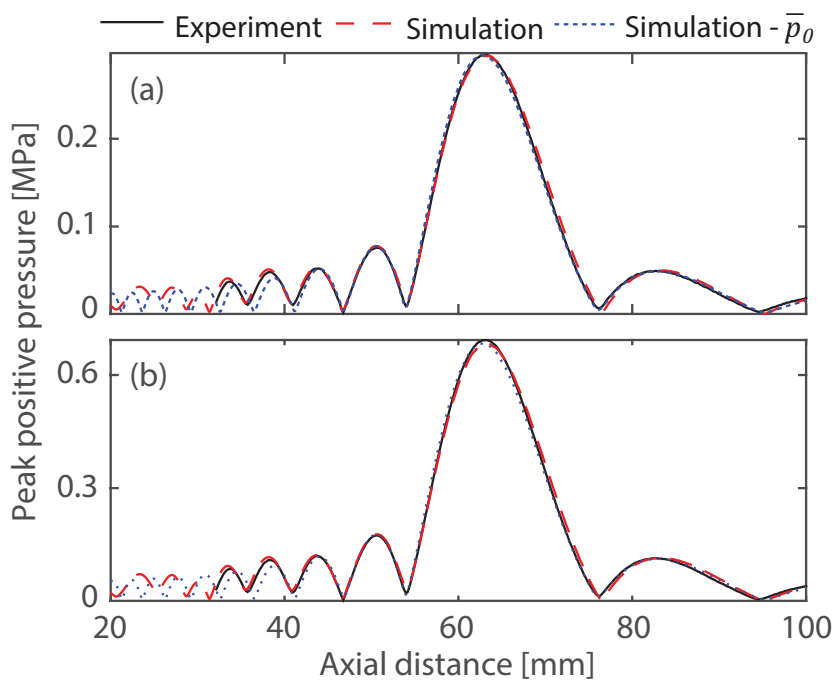

Fig. 8. Comparison of measured and simulated axial pressures under continuous wave conditions at drive voltages of (a) $2.7 \mathrm{~V}_{\text {rms }}$, and (b) 5.4 $\mathrm{V}_{\text {rms. }}$. The red dashed lines show the simulation results using the pressure signal at each grid point of the bowl model calculated using holography, and the blue dotted lines show the simulation results using k-Wave with uniformly radiating mass sources distributed across the transducer surface.

sinusoidal toneburst signals which were applied as the source pressure within $\mathrm{k}$-Wave. In the second set of simulations, a single sinusoidal toneburst was applied as a mass source at all grid points forming the bowl surface $\left(\bar{p}_{0}\right)$. The amplitude 

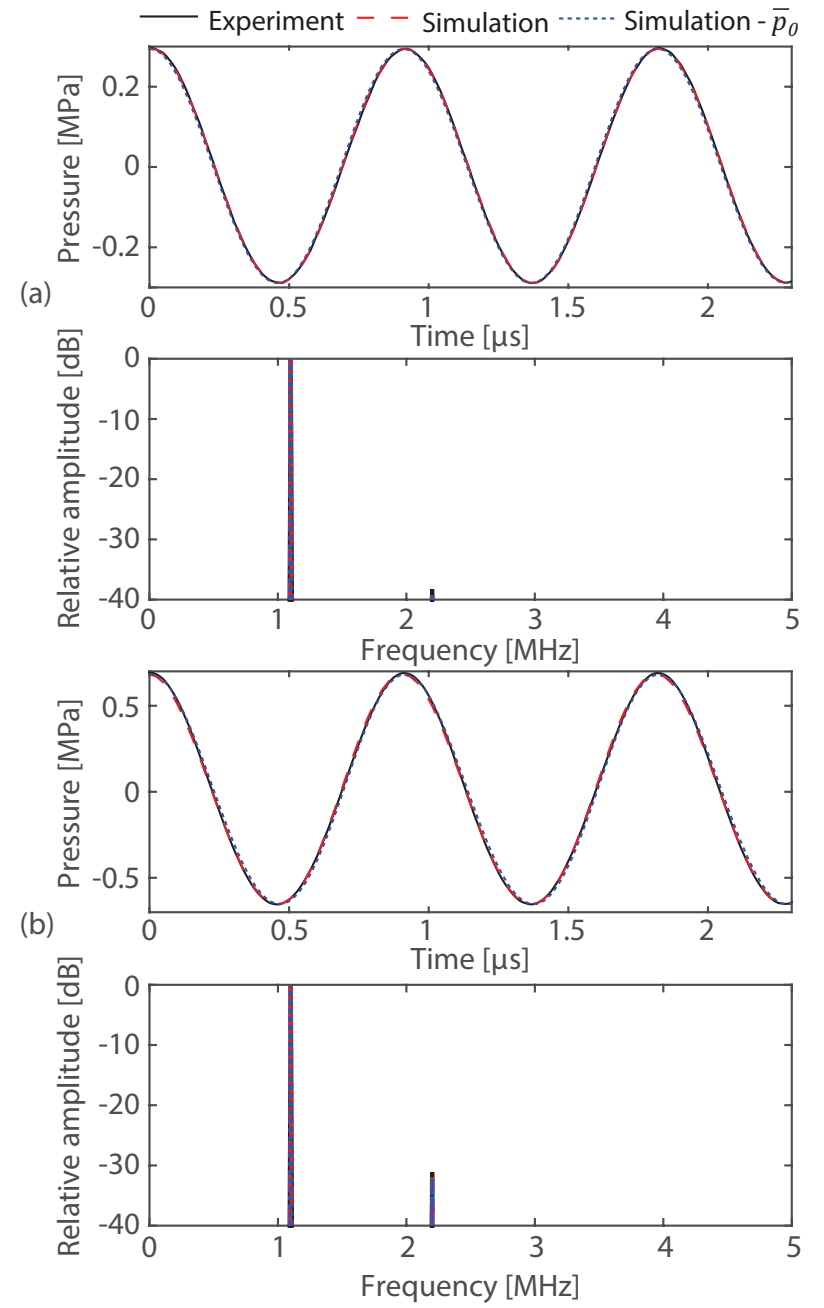

Fig. 9. Comparison of measured and simulated steady state focal waveforms and frequency spectra under continuous wave conditions at drive voltages of (a) $2.7 \mathrm{~V}_{\text {rms }}$ and (b) $5.4 \mathrm{~V}_{\text {rms }}$.

of this signal was derived from the mean pressure magnitude over the bowl surface and an additional scaling factor derived from the measured axial pressure.

The simulations were performed using a grid discretisation of $10.8 \mathrm{PPW}$ ( $125 \mu \mathrm{m}$ point spacing), with a grid size of 864 $\times 648 \times 648$ grid points. Scaling to correct for the effects of source staircasing was performed as discussed in Sec. II-C. For comparisons at higher drive levels, the source pressure was additionally scaled by the increase in drive voltage. The simulated pressure was obtained in a time window which occurred after the field had reached steady state.

A comparison of the pressure field simulated using $\mathrm{k}$-Wave with the pressure field calculated from the source hologram using the Rayleigh integral is shown in Fig. 7. The axial and lateral planes through the focus simulated by the two models show very good agreement. The measured and simulated axial pressure distributions at both drive levels are shown in Fig. 8. There is excellent agreement between the measured axial profile and the simulations using the discrete bowl model in both the focal region and also in the near field. A comparison of the measured and simulated focal waveforms and their spectra at both drive levels is shown in Fig. 9. Scaling of the source pressure by the drive voltage achieves excellent agreement with measurements made at this drive level.

When a uniform mass source distribution is used, there is still excellent agreement in the focal region as can be seen in the axial profiles and the focal waveforms. Agreement is also good in the first pre and post focal maxima but becomes worse in the near field, with the maxima appearing at different axial locations. This is consistent with fields calculated by other models with a uniform distribution of normal vibrational velocity at the source surface $[16,17]$. These differences in the near field are due to the absence of Lamb waves in the source pressure distribution when a uniform source vibration is assumed. Lamb waves are plate waves generated due to the edges of the transducer being fixed. They propagate towards the centre of the source and as the source is coupled to a liquid medium some energy is radiated into this medium $[16,18]$. The effect of the Lamb waves is to make the source surface vibration non-uniform. This is visible as rings on the source hologram shown in Fig. 6(a).

\section{Pulsed Wave Conditions}

For the pulsed wave experiments, the source pressure distribution was obtained by projecting the measured pressure waveforms back to the grid points forming the discrete bowl source using k-Wave (this could equally be done using a transient Rayleigh integral code or similar). The pressure was again also projected onto an area outside the dimensions of the transducer. The peak positive pressure on the bowl surface is shown in Fig. 6(b). Analogous to the continuous wave case, two sets of simulations were run with different drive conditions. In the first set of simulations, the time domain signals at each grid point on the source obtained via back projection of the measured data were used directly as the source pressure within k-Wave. In the second set, the waveform from the central grid point of the discrete bowl source was scaled to the mean peak positive pressure across the transducer, and this was used to define the mass source applied to all grid points on the discrete bowl source $\bar{p}_{0}$. Again an additional scaling factor derived from the measured axial pressure was used in the $\bar{p}_{0}$ case.

The simulations were performed using a grid discretisation of $9.3 \mathrm{PPW}$ (150 $\mu \mathrm{m}$ point spacing), with a grid size of 768 $\times 576 \times 576$ grid points. Correction for the effect of source staircasing was again performed as previously described. For comparison with measurements made at higher drive levels, the source pressure was also scaled by the increase in drive voltage.

The axial pressure profiles for both drive levels are shown in Fig. 10. Agreement between the measured and simulated axial pressure is excellent for both source pressure conditions. The effect of the uniform source distribution on the near field is far less significant than in the quasi steady state case. There are some small differences in the length of the prefocal lobes, but their amplitude and position is not significantly different from the measured data. This is because in the pulsed wave case, the Lamb waves are not temporally coincident with the main pulse except near the edge of the transducer, so they 


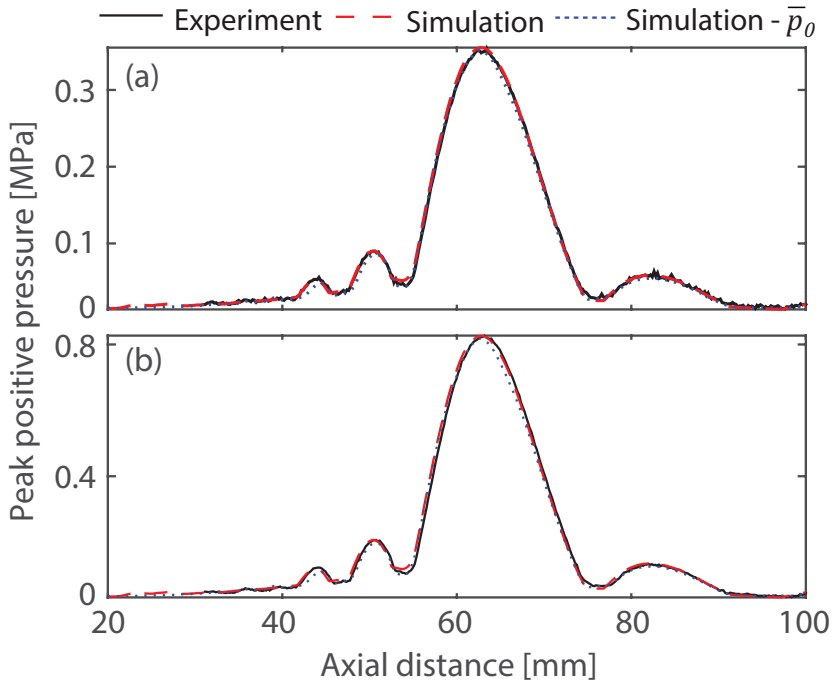

Fig. 10. Comparison of measured and simulated axial pressures under pulsed wave conditions at drive voltages of (a) $7.3 \mathrm{~V}_{\mathrm{pp}}$ and (b) $16.6 \mathrm{~V}_{\mathrm{pp}}$.

have little effect on the axial peak positive pressure profile. There is also excellent agreement between the measured and simulated focal waveforms shown in Fig. 11. There are some minor differences in the shape of the waveforms in the $\bar{p}_{0}$ case, which likely arise from features present in the single source waveform that was applied to all source grid points.

\section{SUMMARY}

A method for generating a discrete bowl shape on a regular Cartesian grid is presented. This can be used to directly model bowl shaped transducers in ultrasound simulations based on a Cartesian grid in place of planar boundary conditions. The functions (called makeBowl, makeMultiBowl, makeArc and makeMultiArc) will be made available with the next release of the open-source k-Wave toolbox [10].

The acoustic fields generated using these source geometries agree well with the fields predicted by the O'Neil solution, the Rayleigh integral, and measurements of the field of a spherically focused transducer. Agreement between the simulated and measured acoustic fields was demonstrated using low transducer drive levels in order to allow the use of a conventional PVDF hydrophone. Although the focal pressures were much lower than those used for HIFU treatments, the validity of the model of source geometry holds regardless of the driving source pressure. A uniform mass source distribution applied to the grid points of the discrete bowl model can be used to accurately predict the pressure in the focal region, but the full source pressure distribution is required in order to correctly simulate the near field, particularly for continuous wave excitation. This is due to the presence of Lamb waves, which propagate across the transducer surface creating a nonuniform source pressure. Accurate source holograms could be obtained from measurements provided the pressure can be measured over an appropriate surface. For complex transducer geometries where this is not possible (e.g., a hemispherical array), a good representation of the pressure in the focal volume could be obtained simply using a set of discrete
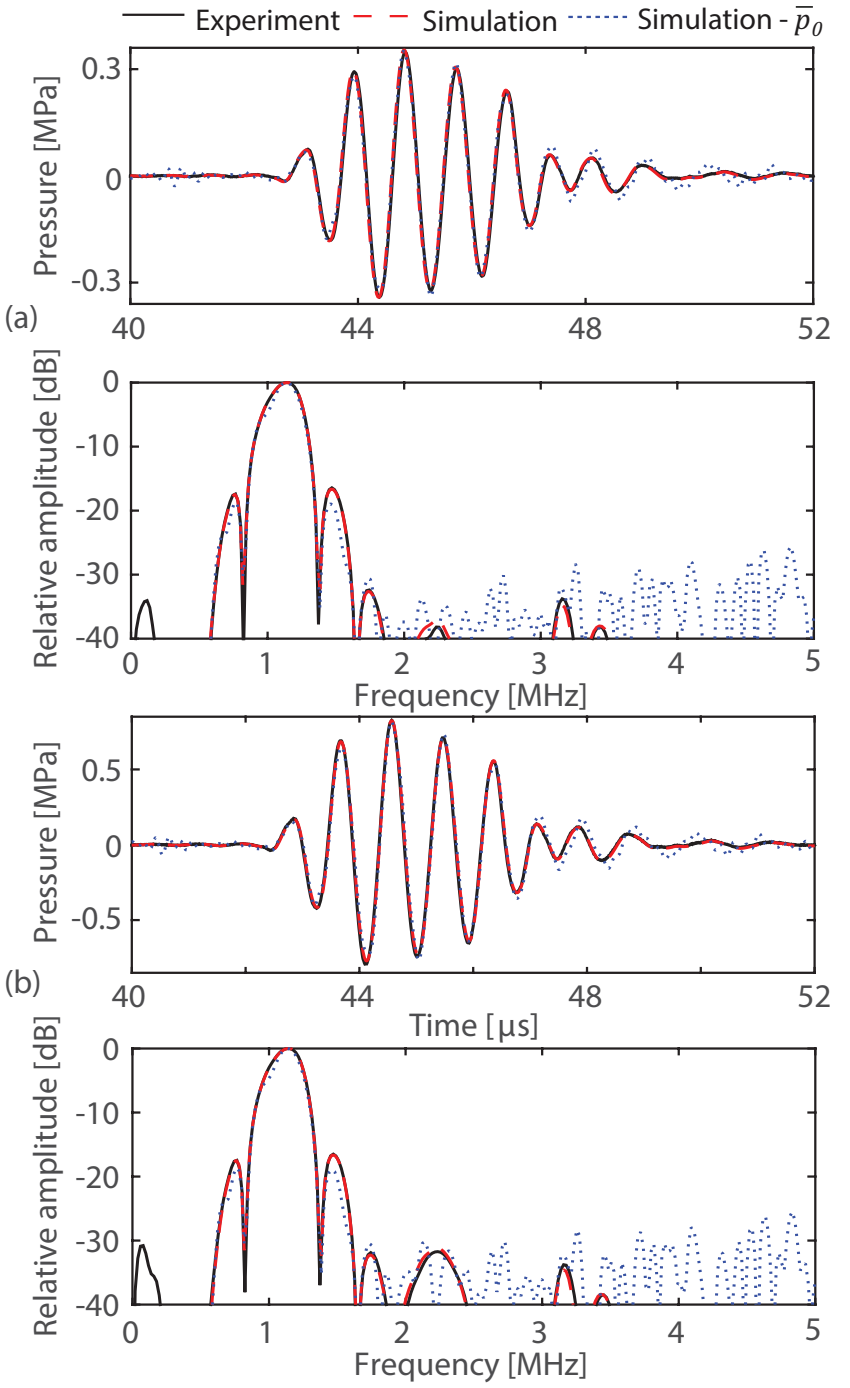

Fig. 11. Comparison of measured and simulated focal waveforms and frequency spectra under pulsed wave conditions at input voltages (a) $7.3 \mathrm{~V}_{\mathrm{pp}}$ and (b) $16.6 \mathrm{~V}_{\mathrm{pp}}$.

bowl sources driven by a uniform mass source distribution. In future, the discrete bowl models developed here will be applied to modelling the response of multi-element arrays used in therapeutic ultrasound.

\section{REFERENCES}

[1] P. Gélat, G. ter Haar, and N. Saffari. Modelling of the acoustic field of a multi-element HIFU array scattered by human ribs. Phys. Med. Biol., 56(17):5553-5581, 2011.

[2] W. Kreider, P. Yuldashev, O. A. Sapozhnikov, N. Farr, A. Partanen, M. R. Bailey, and V. A. Khokhlova. Characterization of a multi-element clinical HIFU system using acoustic holography and nonlinear modeling. IEEE Trans. Ultrason. Ferroelectr. Freq. Control, 60(8):16831698, 2013.

[3] O. A. Sapozhnikov, S. A. Tsysar, V. A. Khokhlova, and W. Kreider. Acoustic holography as a metrological tool for characterizing medical ultrasound sources and fields. J. Acoust. Soc. Am., 138(3):1515-1532, 2015. 
[4] M. S. Canney, M. R. Bailey, L. A. Crum, V. A. Khokhlova, and O. A. Sapozhnikov. Acoustic characterization of high intensity focused ultrasound fields: A combined measurement and modeling approach. $J$. Acoust. Soc. Am., 124(4):2406-2420, 2008.

[5] X. Zeng and R. J. McGough. Optimal simulations of ultrasonic fields produced by large thermal therapy arrays using the angular spectrum approach. J. Acoust. Soc. Am., 125(5):2967-2977, 2009.

[6] G. T. Clement, J. Sun, T. Giesecke, and K. Hynynen. A hemisphere array for non-invasive ultrasound brain therapy and surgery. Phys. Med. Biol., 45(12):3707-19, 2000.

[7] M. D. Verweij, B. E. Treeby, K. W. A. van Dongen, and L. Demi. Simulation of Ultrasound Fields. In A. Brahme, editor, Comprehensive Biomedical Physics, volume 2, chapter 2.19, 465-500. Elsevier, Amsterdam, 2014.

[8] J. Van Aken and M. Novak. Curve-drawing algorithms for raster displays. ACM Transactions on Graphics, 4(2): 147-169, 1985.

[9] E. Andres. Discrete circles, rings and spheres. Computers \& Graphics, 18(5):695-706, 1994.

[10] B. E. Treeby and B. T. Cox. k-Wave: MATLAB toolbox for the simulation and reconstruction of photoacoustic wave fields. J. Biomed. Opt., 15(2):021314, 2010.

[11] B. E. Treeby, J. Jaros, A. P. Rendell, and B. T. Cox. Modeling nonlinear ultrasound propagation in heterogeneous media with power law absorption using a k-space pseudospectral method. J. Acoust. Soc. Am., 131(6): 4324-4336, 2012.

[12] J. Jaros, A. P. Rendell, and B. E. Treeby. Full-wave nonlinear ultrasound simulation on distributed clusters with applications in high-intensity focused ultrasound. Int. J. High Perf. Comput. Appl., 2015.

[13] R. S. C. Cobbold. Foundations of Biomedical Ultrasound. Oxford University Press, New York, 2007.

[14] J. L. Robertson, B. T. Cox, and B. E. Treeby. Quantifying numerical errors in the simulation of transcranial ultrasound using pseudospectral methods. In IEEE International Ultrasonics Symposium, 2000-2003, 2014.

[15] H. T. O'Neil. Theory of focusing radiators. J. Acoust. Soc. Am., 21(5):516-526, 1949.

[16] D. Cathignol, O. A. Sapozhnikov, and J. Zhang. Lamb waves in piezoelectric focused radiator as a reason for discrepancy between O'Neil's formula and experiment. J. Acoust. Soc. Am., 101(3):1286-1297, 1997.

[17] O. V. Bessonova and V. Wilkens. Membrane hydrophone measurement and numerical simulation of HIFU fields up to developed shock regimes. IEEE Trans. Ultrason. Ferroelectr. Freq. Control, 60(2):290-300, 2013.

[18] O. A. Sapozhnikov and M. A. Smagin. Finding the dispersion relations for lamb-type waves in a concave piezoelectric plate by optical visualization of the ultrasound field radiated into a fluid. Acoustical Physics, 61 (2):181-187, 2015. 